\title{
Questioning the Affinity of Electrophilic Astatine for Sulfur- containing Compounds: Unexpected Bindings Revealed
}

Fadel Bassal, ${ }^{a}$ Julie Champion, ${ }^{b}$ Sylvain Pardoue, ${ }^{a, b}$ Mahamadou Seydou, ${ }^{c}$ Andrea Sabatié-Gogova, ${ }^{b}$ David Deniaud, ${ }^{a}$ Jean-Yves Le Questel, ${ }^{a}$ Gilles Montavon, ${ }^{b, *}$ and Nicolas Galland ${ }^{a, *}$

a Université de Nantes, CNRS, CEISAM UMR 6230, F-44000 Nantes (France).

b IMT Atlantique, CNRS, SUBATECH UMR 6457, F-44307 Nantes (France).

c Université de Paris, CNRS, ITODYS UMR 7086, 15 rue J.A. de Baïf, F-75013 Paris (France).

\section{Supporting Information}

I. Thermodynamic models used to derive the values of the complexation constants S2

Case 1: only the species $\mathrm{AtO}^{+}$is present in the system. S2

Case 2: complexation of $\mathrm{AtO}^{+}$with aniline $(\mathrm{L}=\mathrm{An})$ in the presence of $\mathrm{AtO}(\mathrm{OH})$. S3

II. Assessment of the stability for the studied ligands 54

Table S1. Stability results. S4

III. Computed structures at the B3LYP/mAVDZ level of theory S5

Geometries of the conformers displayed on Figures 3, 4 and 5. S5

Supplementary structures of complexes with thiourea, thioacetamide, 2-(thiophen-2-yl)acetic acid and aniline. S7 


\section{Thermodynamic models used to derive the values of the complexation constants}

The analytical expressions used for the determination of the complexation constants in aqueous phase $\left(K_{\mathrm{L}} \equiv \beta_{\mathrm{AtOL}^{+}}\right)$are derived below. The different cases encountered are presented, from the simplest to the most complicated.

\section{Case 1: only the species $\mathrm{AtO}^{+}$is present in the system.}

All the expressions are derived based on the different equilibria and associated constants given below:

$$
\begin{aligned}
& \mathrm{AtO}_{a q}^{+} \rightleftarrows A t O_{o r g}^{+} ; D_{\mathrm{AtO}^{+}}=\frac{\left[\mathrm{AtO}^{+}\right]_{\text {org }}}{\left[\mathrm{AtO}^{+}\right]_{a q}} \\
& \mathrm{AtOL}_{a q}^{+} \rightleftarrows A t O L_{o r g}^{+} ; D_{\mathrm{AtOL}^{+}}=\frac{\left[\mathrm{AtOL}^{+}\right]_{\text {org }}}{\left[\mathrm{AtOL}^{+}\right]_{a q}} \\
& \mathrm{AtO}_{a q}^{+}+L_{a q} \rightleftarrows A t O L_{a q}^{+} ; \beta_{\mathrm{AtOL}^{+}}=\frac{\left[\mathrm{AtOL}^{+}\right]_{a q}}{\left[\mathrm{AtO}^{+}\right]_{a q}[\mathrm{~L}]_{a q}} \\
& \text { AtOL L }_{a q}^{+}+L_{a q} \rightleftarrows A t O L_{2 a q}^{+} ; \beta_{\mathrm{AtOL}_{2}}{ }^{+}=\frac{\left[\mathrm{AtOL}_{2}^{+}\right]_{a q}}{\left[\mathrm{AtOL}^{+}\right]_{a q}[L]_{a q}}
\end{aligned}
$$

With $D_{\mathrm{AtO}^{+}}$the distribution coefficient of $\mathrm{AtO}^{+}$between aqueous and organic phases (usually very low value), $D_{\mathrm{AtOL}^{+}}$the distribution coefficient of the $1: 1$ complex and $\beta_{\mathrm{AtOL}^{+}}$and $\beta_{\mathrm{AtOL}_{2}}$ the complexation constants leading to $1: 1$ and $1: 2 \mathrm{AtO}^{+}: \mathrm{L}$ adducts, respectively.

A 1:1 complex is formed exclusively in the aqueous phase:

$$
D=\frac{D_{A t O^{+}}}{1+\beta_{A t O L^{+}}[L]_{a q}}
$$

1:1 and 1:2 complexes are formed in the aqueous phase:

$$
D=\frac{D_{\text {AtO }^{+}}}{1+\beta_{\text {AtOL }^{+}}[L]_{a q}+\beta_{\text {AtOL }^{+}} \beta_{\text {AtOL }_{2}}+[L]_{a q}{ }^{2}}
$$

A 1:1 complex is formed in the aqueous phase and it is partially soluble in the organic phase: 


$$
D=\frac{D_{A^{+} O^{+}}+D_{A^{\prime O L} L^{+}} \beta_{A_{t O L^{+}}}[L]_{a q}}{1+\beta_{A t O L^{+}}[L]_{a q}}
$$

\section{Case 2: complexation of $\mathrm{AtO}^{+}$with aniline $(\mathrm{L}=\mathrm{An})$ in the presence of $\mathrm{AtO}(\mathrm{OH})$.}

All the expressions are derived based on the equilibria and associated constants previously presented, and those given below:

$$
\begin{aligned}
& A n_{a q} \rightleftarrows A n_{o r g} ; D_{A n}=\frac{[A n]_{o r g}}{[A n]_{a q}} \\
& A n H_{(a q)}^{+} \rightleftarrows A n_{(a q)}+H_{(a q)}^{+} ; K_{a 2}=\frac{[A n]_{a q}\left[H^{+}\right]_{a q}}{\left[A n H^{+}\right]_{a q}} \\
& A t O_{(a q)}^{+}+H_{2} O \quad \rightleftarrows A t O(O H)_{(a q)}+H_{(a q)}^{+} ; K_{a}=\frac{[A t O(O H)]_{a q}\left[H^{+}\right]_{a q}}{\left[A t O^{+}\right]_{a q}} \\
& {[A n]_{\text {total }}=[A n]_{a q}+\left[A n H^{+}\right]_{a q}} \\
& {[A n]_{a q}=[A n]_{\text {total }} * \frac{\frac{K_{a 2}}{\left[H^{+}\right]_{a q}}}{1+\frac{K_{a 2}}{\left[H^{+}\right]_{a q}}}}
\end{aligned}
$$

Where $D_{A n}$ is the ligand distribution between organic and aqueous phase, $[A n]_{\text {total }}$ is the total concentration of aniline in aqueous phase, $\left[\mathrm{AnH}^{+}\right]_{a q}$ is the concentration of protonated aniline (nonreactive one), and $[A n]_{a q}$ is the concentration of non-protonated aniline (reactive one).

$$
D=D_{A n}+\frac{D_{A t O(O H)}+D_{A t O A n^{+}}+\beta_{A t O A n^{+}} \frac{\left[H^{+}\right]_{a q}}{K_{a}}[A n]_{a q}}{1+\beta_{A t O A n^{+}}+\frac{\left[H^{+}\right]_{a q}}{K_{a}}[A n]_{a q}}
$$




\section{Assessment of the stability for the studied ligands}

The $R_{f}$ values obtained by TLC for the ligands in acid oxidizing solution were compared with those obtained in methanol. The results show that the $R_{f}$ values for each ligand are close to each other and do not move over time, showing a relative stability in the time scale required to establish the complexation equilibria $(\sim 2 \mathrm{~h})$. The mass spectra were recorded for ligands dissolved at high concentrations $\left(\sim 10^{-1} \mathrm{M}\right)$ in solutions both oxidizing and acidic. Positive-ESIMS revealed $[\mathrm{M}]^{+}$molecular peak, i.e. $60.3,59.3,75.1$ and 142.9 as the peaks characterizing urea, acetamide, thioacetamide and 2thiophen-2-yl)acetic acid, respectively (Table S1). For thiourea, the $[\mathrm{M}]^{+}$has been detected at $\mathrm{m} / \mathrm{z} 76.1$, whereas the most abundant ion has appeared at $\mathrm{m} / \mathrm{z}$ 107.9. Such a species being not observed from TLC analysis, one can imagine that it could be produced in the source plasma of the spectrometer and could be assigned to a product resulting from the oxidation of thiourea. Taking into account the context of the article, an extensive study has not been conducted. Furthermore, thiourea has already been studied in the past under similar conditions, ${ }^{14}$ and therefore serves as a reference in the present work. Based on these considerations, we decided to keep it in the experimental part of the proposed study.

(14) Dreyer, R.; Dreyer, I.; Fischer, S.; Hartmann, H.; Roesch, F. Synthesis and characterization of cationic astatine compounds with sulfurcontaining ligands stable in aqueous solutions. J. Radioanal. Nucl. Chem. 1985, 96, 333-341.

Table S1. Stability results.

\begin{tabular}{lll} 
Ligand & $\mathbf{R}_{\mathbf{f}}$ (solvent) by TLC & Molar mass by ESIMS \\
\hline Thiourea & 0.81 (methanol-toluene, 7:3) & $76.1,107.9$ \\
Thioacetamide & $0.22\left(\right.$ dipe $^{a}$-toluene, 1:1) & 75.1 \\
2-(thiophen-2-yl)acetic acid & $b$ & 142.9 \\
Urea & 0.56 (methanol-toluene, 7:3) & 60.3 \\
Acetamide & 0.72 (methanol-toluene, 7:3) & 59.3 \\
${ }^{a}$ Diisopropyl ether. ${ }^{b}$ Not done. & &
\end{tabular}




\section{Computed structures at the B3LYP/mAVDZ level of theory}

\section{Geometries of the conformers displayed on Figures 3, 4 and 5.}

Cartesian coordinates (in angstroms).

$\begin{array}{cccc}\mathbf{1} & & & \\ \text { S } & 1.7961 & -1.1269 & 0.1223 \\ \text { C } & 2.8514 & 0.2785 & -0.0685 \\ \text { N } & 3.9597 & 0.1484 & -0.7950 \\ \text { N } & 2.5162 & 1.4010 & 0.5445 \\ \text { H } & 4.1549 & -0.7091 & -1.2962 \\ \text { H } & 1.6867 & 1.3898 & 1.1317 \\ \text { H } & 4.6644 & 0.8749 & -0.8356 \\ \text { H } & 3.0634 & 2.2502 & 0.4753 \\ \text { O } & 0.5692 & -0.4630 & 0.9852 \\ \text { AT } & -1.4314 & 0.0209 & -0.0513\end{array}$

\section{2}

$\begin{array}{llll}C & 2.8667 & 0.2765 & -0.0412\end{array}$

$\begin{array}{llll}\text { N } & 2.5581 & 1.4598 & 0.4357\end{array}$

S $\quad 1.7764-1.0368 \quad 0.1509$

$\begin{array}{llll}\mathrm{O} & 0.5461 & -0.3378 & 0.9818\end{array}$

C $4.1637 \quad 0.0050 \quad-0.7303$

AT $\quad-1.4928 \quad-0.0737 \quad-0.0421$

H $\quad 4.2407 \quad-1.0470 \quad-1.0286$

H $1.6792 \quad 1.58390 .9345$

H $\begin{array}{llll}5.0029 & 0.2462 & -0.0621\end{array}$

$\begin{array}{llll}H & 3.1919 & 2.2474 & 0.3536\end{array}$

H $4.24290 .6315 \quad-1.6310$

\section{3}

C $\quad 2.0265 \quad-2.7528 \quad-0.0403$

$\begin{array}{llll}\text { C } & 2.1411 & -1.4276 & 0.7048\end{array}$

S $\quad 2.0861 \quad 0.0000 \quad-0.4546$

$\begin{array}{llll}\text { C } & 2.1411 & 1.4276 & 0.7048\end{array}$

$\begin{array}{llll}\text { C } & 2.0265 & 2.7528 & -0.0403\end{array}$

H $3.1112 \quad-1.3149 \quad 1.2098$

H $\quad 1.3384-1.2725 \quad 1.4389$

$\begin{array}{llll}H & 3.1112 & 1.3149 & 1.2098\end{array}$

$\begin{array}{llll}H & 1.3384 & 1.2725 & 1.4389\end{array}$

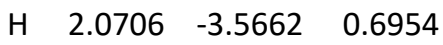

H $2.0706 \quad 3.5663 \quad 0.6954$

H $\quad \begin{array}{llll}1.0752 & -2.8340 & -0.5793\end{array}$

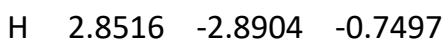

$\begin{array}{llll}H & 2.8516 & 2.8904 & -0.7497\end{array}$

H $\quad 1.0752 \quad 2.8340 \quad-0.5793$

$\begin{array}{llll}\text { O } & 0.6159 & 0.0000 & -1.1192\end{array}$

AT $\quad-1.3769 \quad 0.0000 \quad-0.0002$

$\begin{array}{cccc}\mathbf{4} & & & \\ \text { C } & 4.1050 & -0.5744 & 0.2511 \\ \text { C } & 3.7128 & 0.8606 & 0.6299 \\ \text { C } & 2.6909 & 1.3149 & -0.4127 \\ \text { S } & 1.5703 & -0.1309 & -0.6893 \\ \text { C } & 2.8120 & -1.3555 & -0.0257 \\ \text { O } & 0.5376 & -0.0272 & 0.5742 \\ \text { AT } & -1.7041 & 0.0043 & 0.1486 \\ \text { H } & 2.3252 & -1.7334 & 0.8819 \\ \text { H } & 3.1414 & 1.5084 & -1.3968 \\ \text { H } & 2.9052 & -2.1615 & -0.7619 \\ \text { H } & 2.0498 & 2.1576 & -0.1308 \\ \text { H } & 4.7410 & -0.5722 & -0.6441 \\ \text { H } & 3.2677 & 0.8944 & 1.6328 \\ \text { H } & 4.6655 & -1.0697 & 1.0537 \\ \text { H } & 4.5797 & 1.5332 & 0.6240\end{array}$

\section{5}

$\begin{array}{llll}C & -2.7945 & 1.4012 & -0.3759\end{array}$

C $-3.2398 \quad 0.1224 \quad-0.8384$

S $\quad-2.4832 \quad-1.2187 \quad-0.1957$

C $\quad-1.5020-0.0528 \quad 1.0827$

$\begin{array}{llll}\text { C } & -1.8397 & 1.3188 & 0.5891\end{array}$

H $\quad-2.1462-0.2600 \quad 1.9686$

H $\quad-4.0520-0.0056-1.5586$

$\begin{array}{llll}H & -1.3647 & 2.1777 & 1.0667\end{array}$

$\begin{array}{llll}H & -3.2084 & 2.3299 & -0.7657\end{array}$

$\begin{array}{llll}\mathrm{O} & -0.2461 & -0.4042 & 1.3672\end{array}$

$\begin{array}{llll}\text { AT } & 1.3655 & 0.0477 & -0.1238\end{array}$

\section{6}

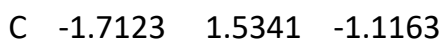

$\begin{array}{llll}\text { C } & -2.0386 & 0.3378 & -0.5670\end{array}$

$\begin{array}{llll}\text { C } & -1.5155 & 0.0940 & 0.8103\end{array}$

$\begin{array}{llll}\text { S } & -0.2835 & 1.5753 & 1.0998\end{array}$

$\begin{array}{llll}\text { C } & -0.8088 & 2.3230 & -0.3181\end{array}$

$\begin{array}{llll}\text { C } & -0.4255 & 3.7075 & -0.7225\end{array}$

$\begin{array}{llll}\text { C } & 0.8654 & 4.2412 & -0.1142\end{array}$

$\begin{array}{llll}\mathrm{O} & 1.4978 & 3.6799 & 0.7497\end{array}$

$\begin{array}{llll}\mathrm{O} & 1.1853 & 5.4163 & -0.6672\end{array}$

$\begin{array}{llll}\text { H } & -0.3807 & 3.7753 & -1.8190\end{array}$

$\begin{array}{llll}\mathrm{H} & -1.2412 & 4.3935 & -0.4288\end{array}$

$\begin{array}{llll}H & 2.0021 & 5.7495 & -0.2553\end{array}$

$\begin{array}{llll}H & -2.0862 & 1.8957 & -2.0736\end{array}$

$\begin{array}{llll}H & -2.6831 & -0.4069 & -1.0362\end{array}$

$\begin{array}{llll}\mathrm{H} & -2.3134 & 0.4013 & 1.5249\end{array}$

$\begin{array}{llll}\text { O } & -1.0767 & -1.1263 & 1.1797\end{array}$

$\begin{array}{llll}\text { AT } & 0.4500 & -2.1375 & -0.0963\end{array}$

$\begin{array}{cccc}\mathbf{7} & & & \\ \mathrm{C} & 2.3278 & -0.0382 & 0.0121 \\ \mathrm{~N} & 2.2205 & 1.2884 & -0.1213 \\ \mathrm{O} & 1.3029 & -0.8105 & 0.1659 \\ \mathrm{~N} & 3.5307 & -0.6283 & -0.0096 \\ \text { AT } & -0.9791 & -0.1573 & 0.2560 \\ \mathrm{O} & -0.7245 & 1.7771 & 0.0330 \\ \mathrm{H} & 3.5830 & -1.6323 & 0.0934 \\ \mathrm{H} & 1.3075 & 1.7381 & -0.1050 \\ \mathrm{H} & 4.3892 & -0.1080 & -0.1252 \\ \mathrm{H} & 3.0373 & 1.8710 & -0.2401\end{array}$

\section{8}

$\begin{array}{llll}\text { C } & 0.6829 & -3.6816 & 0.0971\end{array}$

$\begin{array}{llll}\text { C } & 0.0285 & -2.3340 & 0.0453\end{array}$

$\begin{array}{llll}\mathrm{O} & 0.7905 & -1.3153 & 0.1953\end{array}$

$\begin{array}{llll}\mathrm{N} & -1.2769 & -2.2343 & -0.1477\end{array}$

$\begin{array}{llll}\text { H } & -0.0429 & -4.4910 & -0.0402\end{array}$

$\begin{array}{llll}H & -1.8358 & -3.0699 & -0.2608\end{array}$

$\begin{array}{llll}H & 1.4506 & -3.7349 & -0.6860\end{array}$

$\begin{array}{llll}\text { H } & 1.1869 & -3.7956 & 1.0659\end{array}$

$\begin{array}{llll}\mathrm{H} & -1.7467 & -1.3284 & -0.1871\end{array}$

$\begin{array}{llll}\text { AT } & 0.1446 & 0.9889 & 0.1779\end{array}$

$\begin{array}{llll}\mathrm{O} & -1.7776 & 0.7095 & -0.1211\end{array}$

\section{9}

$\begin{array}{llll}\text { C } & -3.1428 & 0.9303 & -0.2938\end{array}$

$\begin{array}{llll}\text { C } & -3.4883 & -0.5680 & -0.2418\end{array}$

$\begin{array}{llll}\text { C } & -2.1162 & -1.2272 & -0.2236\end{array}$

$\begin{array}{llll}\mathrm{O} & -1.2803 & -0.2950 & 0.5746\end{array}$

$\begin{array}{llll}\text { C } & -1.9223 & 1.0429 & 0.6136\end{array}$

$\begin{array}{llll}\text { H } & -2.1681 & 1.2129 & 1.6694\end{array}$

$\begin{array}{llll}\text { H } & -1.6885 & -1.3076 & -1.2324\end{array}$

$\begin{array}{llll}\text { H } & -1.1846 & 1.7888 & 0.2848\end{array}$

$\begin{array}{llll}\mathrm{H} & -2.0687 & -2.1938 & 0.2887\end{array}$

$\begin{array}{llll}\mathrm{H} & -2.8964 & 1.2406 & -1.3173\end{array}$

$\begin{array}{llll}H & -4.0417 & -0.8132 & 0.6734\end{array}$

$\begin{array}{llll}H & -3.9613 & 1.5638 & 0.0665\end{array}$

$\begin{array}{llll}\text { H } & -4.0781 & -0.8993 & -1.1042\end{array}$

$\begin{array}{llll}\text { AT } & 1.1949 & -0.1611 & 0.0893\end{array}$

$\begin{array}{lllll}\mathrm{O} & 1.1250 & 1.7304 & -0.4087\end{array}$ 
10

$$
\begin{array}{cccc}
\text { C } & 0.0000 & 0.0000 & -3.0977 \\
\text { N } & 0.0000 & 0.0000 & -1.9379 \\
\text { C } & 0.0000 & 0.0000 & -4.5511 \\
\text { H } & 0.7322 & 0.7322 & -4.9174 \\
\text { H } & 0.2680 & -1.0002 & -4.9174 \\
\text { H } & -1.0002 & 0.2680 & -4.9174 \\
\text { AT } & 0.0000 & 0.0000 & 0.7032 \\
\text { O } & 0.0000 & 0.0000 & 2.6707
\end{array}
$$

\section{1}

$\begin{array}{cccc}\mathrm{N} & -0.0974 & 1.7910 & 0.0000 \\ \mathrm{C} & -1.3259 & 2.0923 & 0.0000 \\ \mathrm{C} & 1.0205 & 2.7327 & 0.0000 \\ \mathrm{H} & -1.6597 & 3.1379 & 0.0000 \\ \mathrm{H} & -2.0716 & 1.2789 & 0.0000 \\ \mathrm{H} & 0.6489 & 3.7662 & 0.0000 \\ \mathrm{H} & 1.6344 & 2.5621 & 0.8935 \\ \mathrm{H} & 1.6344 & 2.5621 & -0.8935 \\ \text { AT } & 0.1816 & -0.7050 & 0.0000 \\ \mathrm{O} & -1.7800 & -0.8374 & 0.0000\end{array}$

\section{2}

$\begin{array}{llll}\text { C } & 0.7587 & -3.1577 & -0.1340\end{array}$

$\begin{array}{llll}C & -0.6726 & -2.6763 & -0.3149\end{array}$

$\begin{array}{llll}\text { N } & -0.9662 & -1.4959 & 0.5451\end{array}$

$\begin{array}{llll}\text { AT } & 0.2791 & 0.6905 & 0.0159\end{array}$

$\begin{array}{llll}\text { O } & -1.3486 & 1.7291 & -0.3240\end{array}$

$\begin{array}{llll}\mathrm{H} & -0.8243 & -1.7074 & 1.5356\end{array}$

$\begin{array}{llll}\text { H } & -1.9288 & -1.1659 & 0.4327\end{array}$

$\begin{array}{llll}\mathrm{H} & -1.3864 & -3.4716 & -0.0473\end{array}$

H $\quad-0.8779-2.3848 \quad-1.3525$

H $\quad 0.9301 \quad-4.0460-0.7541$

H $\quad 1.4894 \quad-2.3964 \quad-0.4489$

$\begin{array}{llll}H & 0.9655 & -3.4356 & 0.9092\end{array}$

13

$\begin{array}{llll}\text { H } & 2.2816 & 2.1966 & 0.2662\end{array}$

$\begin{array}{llll}C & 1.2913 & 2.1300 & -0.1974\end{array}$

$\begin{array}{llll}\mathrm{N} & 0.3842 & 1.3082 & 0.6827\end{array}$

$\begin{array}{llll}\text { C } & 0.5501 & 3.4639 & -0.3506\end{array}$

$\begin{array}{llll}\text { C } & -0.9889 & 1.8963 & 0.6712\end{array}$

$\begin{array}{llll}\text { C } & -0.9265 & 3.0346 & -0.3587\end{array}$

$\begin{array}{llll}\mathrm{H} & 0.7516 & 1.2264 & 1.6291\end{array}$

H $\quad 1.3916 \quad 1.6306 \quad-1.1696$

$\begin{array}{llll}H & 0.8531 & 3.9880 & -1.2639\end{array}$

$\begin{array}{cccc}\mathrm{H} & 0.7624 & 4.1187 & 0.5054\end{array}$

$\begin{array}{llll}H & -1.7260 & 1.1124 & 0.4087\end{array}$

$\begin{array}{llll}\mathrm{H} & -1.2209 & 2.2408 & 1.6877\end{array}$

$\begin{array}{llll}H & -1.6097 & 3.8471 & -0.0882\end{array}$

$\begin{array}{llll}H & -1.2208 & 2.6683 & -1.3512\end{array}$

$\begin{array}{llll}\text { AT } & 0.1298 & -1.1370 & -0.0502\end{array}$

$\begin{array}{llll}\text { O } & -1.8074 & -0.8528 & -0.2705\end{array}$

\section{4}

$\begin{array}{llll}\text { N } & 1.2179 & 4.4188 & 0.0000\end{array}$

$\begin{array}{llll}\text { C } & 0.4495 & 3.3406 & 0.0000\end{array}$

$\begin{array}{llll}\text { C } & 0.0451 & 2.7489 & 1.2549\end{array}$

$\begin{array}{llll}\text { C } & -0.7690 & 1.6690 & 1.2554\end{array}$

$\begin{array}{llll}\text { C } & -1.3621 & 1.1031 & 0.0000\end{array}$

$\begin{array}{llll}\text { C } & -0.7690 & 1.6690 & -1.2554\end{array}$

$\begin{array}{llll}\text { C } & 0.0451 & 2.7489 & -1.2549\end{array}$

$\begin{array}{llll}\text { H } & 1.5251 & 4.8504 & 0.8641\end{array}$

$\begin{array}{llll}H & 1.5251 & 4.8504 & -0.8641\end{array}$

$\begin{array}{llll}H & 0.4245 & 3.1750 & 2.1845\end{array}$

$\begin{array}{llll}\mathrm{H} & -1.0704 & 1.1940 & 2.1905\end{array}$

$\begin{array}{llll}\text { H } & -2.4068 & 1.5114 & 0.0000\end{array}$

$\begin{array}{llll}\text { H } & -1.0704 & 1.1940 & -2.1905\end{array}$

$\begin{array}{llll}\text { H } & 0.4245 & 3.1750 & -2.1845\end{array}$

$\begin{array}{llll}\mathrm{O} & -1.5443 & -0.2843 & 0.0000\end{array}$

$\begin{array}{llll}\text { AT } & 0.2874 & -1.5324 & 0.0000\end{array}$

\section{5}

$\begin{array}{llll}\mathrm{H} & -1.2125 & 2.2543 & -1.7631\end{array}$

$\begin{array}{llll}\mathrm{N} & -0.5979 & 2.3476 & -0.9588\end{array}$

$\begin{array}{llll}\text { C } & -0.9141 & 1.7572 & 0.3951\end{array}$

$\begin{array}{llll}\text { C } & 0.4876 & 3.0650 & -0.9111\end{array}$

$\begin{array}{llll}\text { C } & 0.3146 & 2.1874 & 1.1657\end{array}$

$\begin{array}{llll}\text { C } & 1.0970 & 2.9869 & 0.4021\end{array}$

$\begin{array}{llll}\text { H } & -1.7594 & 2.3778 & 0.7698\end{array}$

$\begin{array}{llll}H & 0.8409 & 3.6383 & -1.7692\end{array}$

$\begin{array}{llll}H & 0.4632 & 1.9134 & 2.2083\end{array}$

H $\quad 2.0172 \quad 3.4934 \quad 0.6795$

$\begin{array}{llll}\text { O } & -1.3370 & 0.4670 & 0.3810\end{array}$

$\begin{array}{llll}\text { AT } & 0.1504 & -1.1717 & 0.0494\end{array}$

\section{6}

$\begin{array}{llll}\mathrm{N} & 1.2179 & 4.4188 & 0.0000\end{array}$

$\begin{array}{llll}\text { C } & 0.4495 & 3.3406 & 0.0000\end{array}$

$\begin{array}{llll}\text { C } & 0.0451 & 2.7489 & 1.2549\end{array}$

$\begin{array}{llll}\text { C } & -0.7690 & 1.6690 & 1.2554\end{array}$

$\begin{array}{llll}\text { C } & -1.3621 & 1.1031 & 0.0000\end{array}$

$\begin{array}{llll}\text { C } & -0.7690 & 1.6690 & -1.2554\end{array}$

$\begin{array}{llll}\text { C } & 0.0451 & 2.7489 & -1.2549\end{array}$

$\begin{array}{llll}\text { H } & 1.5251 & 4.8504 & 0.8641\end{array}$

$\begin{array}{llll}H & 1.5251 & 4.8504 & -0.8641\end{array}$

$\begin{array}{llll}H & 0.4245 & 3.1750 & 2.1845\end{array}$

$\begin{array}{llll}\text { H } & -1.0704 & 1.1940 & 2.1905\end{array}$

$\begin{array}{llll}\mathrm{H} & -2.4068 & 1.5114 & 0.0000\end{array}$

$\begin{array}{llll}\mathrm{H} & -1.0704 & 1.1940 & -2.1905\end{array}$

$\begin{array}{llll}H & 0.4245 & 3.1750 & -2.1845\end{array}$

$\begin{array}{llll}\mathrm{O} & -1.5443 & -0.2843 & 0.0000\end{array}$

$\begin{array}{llll}\text { AT } & 0.2874 & -1.5324 & 0.0000\end{array}$ 


\section{Supplementary structures of complexes with thiourea, thioacetamide, 2- (thiophen-2-yl)acetic acid and aniline.}

Geometries (cartesian coordinates in angstroms) of other low lying conformers, i.e. their relative stabilities in solution $\left(\Delta G_{298}^{*}\right)$ with respect to the most stable conformer displayed on Figures 3 or 5 , are no more than $6 \mathrm{kcal} \mathrm{mol}^{-1}$.

\section{Complexes with thiourea}

At-S interaction, $\Delta G_{298}^{*}=+6.0 \mathrm{kcal} \mathrm{mol}^{-1}$
$\begin{array}{cccc}\text { S } & 1.3952 & -1.1914 & 0.3608 \\ \text { C } & 2.4349 & 0.1723 & -0.0629 \\ \text { N } & 3.6993 & -0.1437 & -0.3578 \\ \text { N } & 2.0220 & 1.4257 & -0.0537 \\ \text { H } & 3.9865 & -1.1111 & -0.4282 \\ \text { H } & 1.0433 & 1.6826 & 0.1945 \\ \text { H } & 4.4124 & 0.5670 & -0.4763 \\ \text { H } & 2.6466 & 2.1724 & -0.3385 \\ \text { AT } & -1.1679 & -0.1178 & 0.1521 \\ \text { O } & -0.6477 & 1.7812 & 0.4438\end{array}$

\section{Complexes with thioacetamide}

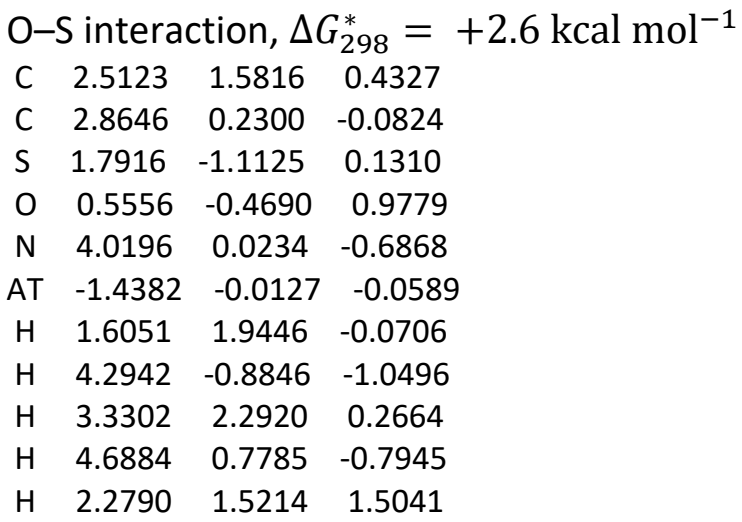

At-S interaction, $\Delta G_{298}^{*}=+5.7 \mathrm{kcal} \mathrm{mol}^{-1}$ C $-2.4756 \quad 0.1825 \quad 0.0725$

$\begin{array}{llll}\mathrm{N} & -2.0855 & 1.4181 & -0.0680\end{array}$

$\begin{array}{llll}\text { S } & -1.3929 & -1.1782 & 0.1813\end{array}$

$\begin{array}{llll}\text { C } & -3.9331 & -0.1563 & 0.1520\end{array}$

$\begin{array}{llll}\text { AT } & 1.1679 & -0.0757 & -0.0111\end{array}$

$\begin{array}{lllll}O & 0.5859 & 1.8234 & -0.1911\end{array}$

H $\quad-4.2021 \quad-0.8454 \quad-0.6604$

$\begin{array}{llll}\mathrm{H} & -1.0744 & 1.6961 & -0.1275\end{array}$

$\begin{array}{llll}H & -4.5480 & 0.7497 & 0.0763\end{array}$

$\begin{array}{llll}H & -2.7871 & 2.1512 & -0.1230\end{array}$

$\begin{array}{llll}H & -4.1487 & -0.6618 & 1.1034\end{array}$ 


\section{Complexes with 2-(thiophen-2-yl)acetic}

acid

O-C interaction, $\Delta G_{298}^{*}=+0.9 \mathrm{kcal} \mathrm{mol}^{-1}$

$\begin{array}{llll}\text { C } & 1.7051 & -1.5800 & -1.1564\end{array}$

$\begin{array}{llll}\text { C } & 2.0413 & -0.3618 & -0.6607\end{array}$

C $1.5843 \quad-0.0827 \quad 0.7321$

S $\quad 0.3704 \quad-1.5630 \quad 1.1185$

C $0.8493 \quad-2.3504 \quad-0.2935$

$\begin{array}{llll}\text { C } & 0.4533 & -3.7544 & -0.6187\end{array}$

$\begin{array}{llll}\text { C } & -0.9344 & -4.1620 & -0.0924\end{array}$

$\begin{array}{llll}0 & -1.5353 & -3.5106 & 0.7224\end{array}$

$\begin{array}{llll}0 & -1.4353 & -5.3072 & -0.5752\end{array}$

H $\quad 0.5356-3.9121 \quad-1.7034$

H $\quad \begin{array}{llll}\text { H.1871 } & -4.4352 & -0.1493\end{array}$

H $\quad-0.8591 \quad-5.7374 \quad-1.2246$

H $\quad 2.0403 \quad-1.9665 \quad-2.1183$

$\begin{array}{llll}\text { H } & 2.6487 & 0.3773 & -1.1852\end{array}$

H $\quad 2.4136 \quad-0.3775 \quad 1.4160$

$\begin{array}{llll}\text { O } & 1.1625 & 1.1440 & 1.0929\end{array}$

$\begin{array}{llll}\text { AT } & -0.4422 & 2.1133 & -0.1212\end{array}$

O-C interaction, $\Delta G_{298}^{*}=+1.0 \mathrm{kcal} \mathrm{mol}^{-1}$

$\begin{array}{llll}C & -2.2122 & 2.3785 & -0.0776\end{array}$

$\begin{array}{llll}\text { C } & -2.3847 & 1.0358 & -0.0417\end{array}$

$\begin{array}{llll}\text { C } & -1.1623 & 0.2260 & 0.2439\end{array}$

$\begin{array}{llll}\text { S } & 0.2761 & 1.5552 & 0.2339\end{array}$

$\begin{array}{llll}\text { C } & -0.8443 & 2.8046 & 0.0879\end{array}$

$\begin{array}{llll}\text { C } & -0.4986 & 4.2549 & 0.1585\end{array}$

$\begin{array}{llll}\text { C } & 0.9634 & 4.6080 & -0.0780\end{array}$

$\begin{array}{lllll}\text { O } & 1.8554 & 3.7990 & -0.1833\end{array}$

$\begin{array}{llll}\text { O } & 1.1128 & 5.9360 & -0.1420\end{array}$

$\begin{array}{llll}\text { H } & -1.1372 & 4.8170 & -0.5392\end{array}$

$\begin{array}{llll}H & -0.7795 & 4.6298 & 1.1595\end{array}$

$\begin{array}{llll}H & 2.0538 & 6.1433 & -0.2814\end{array}$

$\begin{array}{llll}H & -3.0181 & 3.0984 & -0.2161\end{array}$

$\begin{array}{llll}H & -3.3357 & 0.5248 & -0.1950\end{array}$

$\begin{array}{llll}\text { H } & -1.1550 & -0.0234 & 1.3281\end{array}$

$\begin{array}{llll}0 & -1.0031 & -0.8173 & -0.5986\end{array}$

$\begin{array}{llll}\text { AT } & 0.2729 & -2.5409 & 0.0325\end{array}$

O-C interaction, $\Delta G_{298}^{*}=+1.6 \mathrm{kcal} \mathrm{mol}^{-1}$
$\begin{array}{llll}S & -1.0930 & -1.3790 & 0.7687\end{array}$
$\begin{array}{llll}\text { C } & -0.1148 & -2.2810 & -0.2677\end{array}$
$\begin{array}{llll}\text { C } & 1.2398 & -1.7999 & -0.3860\end{array}$
$\begin{array}{llll}\text { C } & 1.5167 & -0.7269 & 0.3925\end{array}$
$\begin{array}{llll}\text { C } & 0.4394 & -0.2812 & 1.3336\end{array}$
$\begin{array}{llll}\text { C } & -0.6369 & -3.4843 & -0.9764\end{array}$
$\begin{array}{llll}\text { C } & -0.1338 & -4.8215 & -0.3878\end{array}$
$\begin{array}{llll}\text { O } & -0.8519 & -5.7627 & -0.2026\end{array}$
$\begin{array}{llll}0 & 1.1940 & -4.7922 & -0.1516\end{array}$
$\begin{array}{llll}\mathrm{H} & -1.7291 & -3.5263 & -0.9707\end{array}$
$\begin{array}{llll}\text { H } & -0.2825 & -3.4581 & -2.0203\end{array}$

$\begin{array}{cccc}\mathrm{H} & 1.4649 & -5.6689 & 0.1752 \\ \mathrm{H} & 1.9553 & -2.2861 & -1.0461 \\ \mathrm{H} & 2.4804 & -0.2156 & 0.4216 \\ \mathrm{H} & 0.6277 & -0.7523 & 2.3235 \\ \mathrm{O} & 0.1888 & 1.0222 & 1.5276 \\ \text { AT } & -0.0745 & 2.3529 & -0.2513\end{array}$

O-C interaction, $\Delta G_{298}^{*}=+2.0 \mathrm{kcal} \mathrm{mol}^{-1}$

$\begin{array}{llll}\text { C } & -0.5818 & -1.8285 & 1.4112\end{array}$

$\begin{array}{llll}\text { C } & -1.0578 & -0.5715 & 1.5817\end{array}$

$\begin{array}{llll}\text { C } & -1.8078 & 0.0316 & 0.4338\end{array}$

S $\quad-1.5273 \quad-1.3010 \quad-0.9930$

$\begin{array}{llll}\text { C } & -0.7950 & -2.3618 & 0.0893\end{array}$

$\begin{array}{llll}\text { C } & -0.4312 & -3.7560 & -0.3101\end{array}$

$\begin{array}{llll}\text { C } & 1.0433 & -4.0812 & -0.0333\end{array}$

$\begin{array}{llll}\text { O } & 1.7837 & -3.4076 & 0.6415\end{array}$

$\begin{array}{llll}0 & 1.3727 & -5.2266 & -0.6429\end{array}$

$\begin{array}{llll}\text { H } & -1.0311 & -4.4630 & 0.2897\end{array}$

H $\quad-0.6608-3.9581-1.3618$

$\begin{array}{llll}\text { H } & 2.3030 & -5.4325 & -0.4427\end{array}$

$\begin{array}{llll}\text { H } & -0.0741 & -2.4076 & 2.1786\end{array}$

$\begin{array}{llll}H & -0.9802 & -0.0027 & 2.5099\end{array}$

$\begin{array}{llll}\text { H } & -2.8952 & -0.1315 & 0.6018\end{array}$

$\begin{array}{llll}0 & -1.6171 & 1.3115 & 0.0842\end{array}$

AT $\quad 0.4801 \quad 2.0606-0.1460$

O-C interaction, $\Delta G_{298}^{*}=+2.3 \mathrm{kcal} \mathrm{mol}^{-1}$

$\begin{array}{llll}\text { C } & -2.2011 & 2.3874 & -0.1005\end{array}$

$\begin{array}{llll}\text { C } & -2.3770 & 1.0435 & -0.1054\end{array}$

$\begin{array}{llll}C & -1.1706 & 0.2259 & 0.2156\end{array}$

$\begin{array}{llll}\text { S } & 0.2742 & 1.5532 & 0.2834\end{array}$

$\begin{array}{llll}\text { C } & -0.8421 & 2.8049 & 0.1314\end{array}$

$\begin{array}{llll}\text { C } & -0.4832 & 4.2508 & 0.2626\end{array}$

$\begin{array}{llll}C & 0.9711 & 4.5933 & -0.0946\end{array}$

$\begin{array}{lllll}\text { O } & 1.8215 & 3.7514 & -0.2288\end{array}$

$\begin{array}{llll}0 & 1.2565 & 5.8966 & -0.2251\end{array}$

$\begin{array}{llll}\text { H } & -1.1948 & 4.8486 & -0.3256\end{array}$

$\begin{array}{llll}H & -0.6377 & 4.5437 & 1.3176\end{array}$

$\begin{array}{llll}\text { H } & 0.4882 & 6.4743 & -0.1043\end{array}$

$\begin{array}{llll}H & -3.0010 & 3.1108 & -0.2546\end{array}$

$\begin{array}{llll}H & -3.3201 & 0.5386 & -0.3169\end{array}$

H $\quad-1.2026 \quad-0.0292 \quad 1.2986$

$\begin{array}{llll}\mathrm{O} & -0.9825 & -0.8102 & -0.6269\end{array}$

$\begin{array}{llll}\text { AT } & 0.2694 & -2.5420 & 0.0333\end{array}$ 


$\begin{array}{crrr}\mathrm{O} & 0.8743 & 5.9155 & -0.1432 \\ \mathrm{H} & 1.6632 & 3.6045 & 0.9319 \\ \mathrm{H} & 1.8239 & 3.3699 & -0.8226 \\ \mathrm{H} & 1.7908 & 5.8287 & 0.1591 \\ \mathrm{H} & -0.5753 & 2.3654 & -2.0091 \\ \mathrm{H} & -2.0199 & 0.2754 & -1.5135 \\ \mathrm{H} & -2.1660 & 0.7101 & 1.1606 \\ \mathrm{O} & -1.3275 & -1.0784 & 0.8736 \\ \mathrm{AT} & 0.0979 & -2.4126 & -0.2217\end{array}$

O-C interaction, $\Delta G_{298}^{*}=+3.2 \mathrm{kcal} \mathrm{mol}^{-1}$

$\begin{array}{llll}C & -1.4602 & 2.5752 & 0.3626\end{array}$

$\begin{array}{llll}\text { C } & -1.8986 & 1.2952 & 0.3226\end{array}$

$\begin{array}{llll}\text { C } & -0.8771 & 0.2152 & 0.5082\end{array}$

$\begin{array}{llll}\text { S } & 0.8147 & 1.2480 & 0.5575\end{array}$

$\begin{array}{llll}\text { C } & -0.0320 & 2.7021 & 0.5189\end{array}$

$\begin{array}{llll}\text { C } & 0.6782 & 4.0078 & 0.6802\end{array}$

$\begin{array}{llll}\text { C } & 0.3050 & 5.0404 & -0.3939\end{array}$

$\begin{array}{llll}\text { O } & -0.6908 & 5.0037 & -1.0739\end{array}$

$\begin{array}{llll}O & 1.2428 & 5.9955 & -0.4395\end{array}$

$\begin{array}{llll}\text { H } & 0.3762 & 4.4470 & 1.6483\end{array}$

$\begin{array}{llll}H & 1.7653 & 3.8827 & 0.7136\end{array}$

$\begin{array}{llll}H & 0.9793 & 6.6606 & -1.1001\end{array}$

$\begin{array}{llll}H & -2.0978 & 3.4515 & 0.2774\end{array}$

$\begin{array}{llll}H & -2.9396 & 1.0018 & 0.1804\end{array}$

$\begin{array}{llll}H & -0.8919 & -0.1287 & 1.5640\end{array}$

$\begin{array}{llll}\text { O } & -0.9388 & -0.7435 & -0.4289\end{array}$

$\begin{array}{llll}\text { AT } & -0.0597 & -2.7591 & -0.0038\end{array}$

O-C interaction, $\Delta G_{298}^{*}=+3.8 \mathrm{kcal} \mathrm{mol}^{-1}$

$\begin{array}{llll}\text { C } & -0.8329 & -1.8892 & 1.3593\end{array}$

$\begin{array}{llll}\text { C } & -1.3128 & -0.6273 & 1.4711\end{array}$

$\begin{array}{llll}C & -1.8673 & -0.0035 & 0.2268\end{array}$

$\begin{array}{llll}\text { S } & -1.3738 & -1.3131 & -1.1574\end{array}$

$\begin{array}{llll}\text { C } & -0.8364 & -2.4027 & 0.0110\end{array}$

$\begin{array}{llll}\text { C } & -0.4054 & -3.7848 & -0.3511\end{array}$

$\begin{array}{llll}\text { C } & 1.1149 & -3.9682 & -0.1435\end{array}$

$\begin{array}{llll}\text { O } & 1.9035 & -4.0140 & -1.0462\end{array}$

$\begin{array}{llll}0 & 1.4157 & -4.0682 & 1.1669\end{array}$

$\begin{array}{llll}\text { H } & -0.9195 & -4.5010 & 0.3083\end{array}$

$\begin{array}{llll}\text { H } & -0.6213 & -4.0151 & -1.3974\end{array}$

$\begin{array}{llll}\text { H } & 2.3762 & -4.2047 & 1.2527\end{array}$

$\begin{array}{llll}H & -0.4721 & -2.4925 & 2.1901\end{array}$

$\begin{array}{llll}H & -1.3786 & -0.0713 & 2.4080\end{array}$

$\begin{array}{llll}H & -2.9682 & -0.1637 & 0.2219\end{array}$

$\begin{array}{llll}\mathrm{O} & -1.6271 & 1.2833 & -0.0661\end{array}$

$\begin{array}{llll}\text { AT } & 0.4788 & 2.0363 & 0.0022\end{array}$

O-C interaction, $\Delta G_{298}^{*}=+4.0 \mathrm{kcal} \mathrm{mol}^{-1}$
$\begin{array}{llll}\text { C } & -1.4500 & 2.5808 & -0.4399\end{array}$
$\begin{array}{llll}\text { C } & -1.8478 & 1.3195 & -0.1519\end{array}$
$\begin{array}{llll}\text { C } & -0.7755 & 0.3074 & 0.1033\end{array}$
$\begin{array}{lllll}\text { S } & 0.8751 & 1.3319 & -0.3494\end{array}$
$\begin{array}{llll}\text { C } & -0.0221 & 2.7394 & -0.5424\end{array}$

$\begin{array}{cccc}\text { C } & 0.6332 & 4.0637 & -0.7758 \\ \text { C } & 0.2815 & 5.0624 & 0.3526 \\ \text { O } & -0.5555 & 4.8328 & 1.1848 \\ \text { O } & 0.9644 & 6.2174 & 0.3414 \\ \text { H } & 1.7204 & 3.9593 & -0.8775 \\ \text { H } & 0.2449 & 4.4791 & -1.7223 \\ \text { H } & 1.6075 & 6.2795 & -0.3806 \\ \text { H } & -2.1253 & 3.4247 & -0.5632 \\ \text { H } & -2.8879 & 1.0021 & -0.0664 \\ \text { H } & -0.6018 & 0.2288 & 1.1976 \\ \text { O } & -0.9441 & -0.8430 & -0.5596 \\ \text { AT } & 0.0239 & -2.7136 & 0.2060\end{array}$

O-C interaction, $\Delta G_{298}^{*}=+4.3 \mathrm{kcal} \mathrm{mol}^{-1}$

$\begin{array}{llll}C & -0.9031 & 0.2181 & -0.5045\end{array}$

$\begin{array}{llll}\text { S } & 0.7874 & 1.2659 & -0.6135\end{array}$

$\begin{array}{llll}\text { C } & -0.0703 & 2.7115 & -0.5717\end{array}$

$\begin{array}{llll}\text { C } & -1.4924 & 2.5785 & -0.3779\end{array}$

$\begin{array}{llll}\text { C } & -1.9236 & 1.2975 & -0.3095\end{array}$

$\begin{array}{llll}\text { C } & 0.6153 & 4.0306 & -0.7457\end{array}$

$\begin{array}{llll}\text { C } & 0.3395 & 4.9781 & 0.4480\end{array}$

$\begin{array}{llll}\mathrm{O} & 1.1135 & 6.0733 & 0.5063\end{array}$

$\begin{array}{llll}\mathrm{O} & -0.5181 & 4.7686 & 1.2631\end{array}$

$\begin{array}{llll}\text { H } & 1.6930 & 3.8976 & -0.8994\end{array}$

$\begin{array}{llll}H & 0.2064 & 4.5198 & -1.6470\end{array}$

$\begin{array}{llll}H & 1.7664 & 6.1252 & -0.2076\end{array}$

$\begin{array}{llll}H & -2.1351 & 3.4511 & -0.2882\end{array}$

$\begin{array}{llll}\text { H } & -2.9591 & 1.0020 & -0.1349\end{array}$

$\begin{array}{llll}H & -0.9364 & -0.1412 & -1.5545\end{array}$

$\begin{array}{llll}\text { O } & -0.9335 & -0.7233 & 0.4481\end{array}$

$\begin{array}{llll}\text { AT } & -0.0588 & -2.7452 & 0.0384\end{array}$ 


\section{Complexes with aniline}

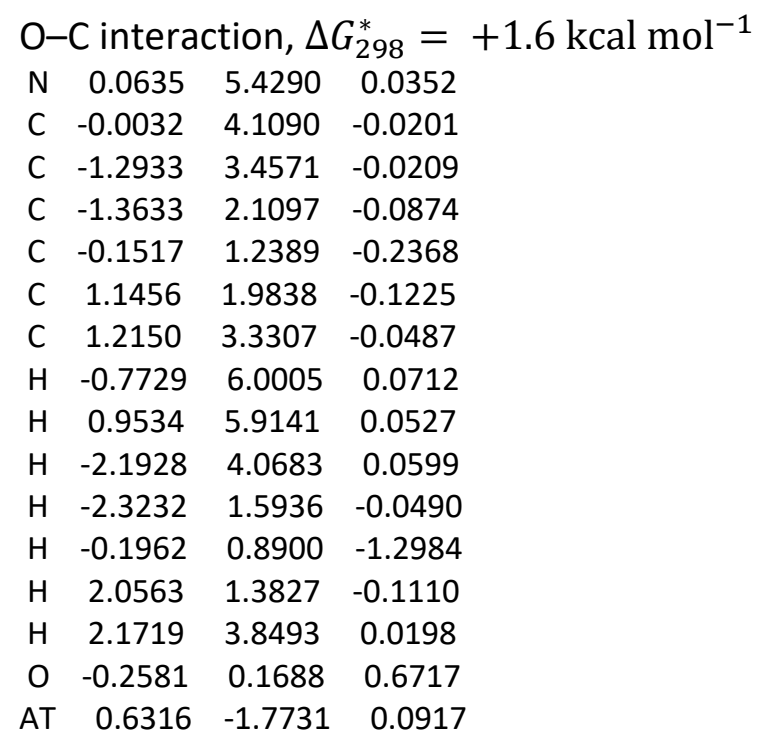

O-C interaction, $\Delta G_{298}^{*}=+2.6 \mathrm{kcal} \mathrm{mol}^{-1}$
$\begin{array}{llll}N & -2.2509 & 1.7136 & 0.0502\end{array}$
C $\quad \begin{array}{llll}-1.0121 & 2.1531 & -0.0019\end{array}$
$\begin{array}{llll}\text { C } & -0.0837 & 1.4425 & -0.9688\end{array}$
C $1.3751 \quad 1.6908 \quad-0.7221$
C $\quad 1.7829 \quad 2.7206 \quad 0.0549$
C $\quad 0.8115 \quad 3.5236 \quad 0.7622$
$\begin{array}{llll}\text { C } & -0.5373 & 3.2657 & 0.7449\end{array}$
H $\quad-2.4953 \quad 0.8805 \quad-0.4799$
$\begin{array}{llll}H & -2.9644 & 2.1680 & 0.6083\end{array}$
H $\quad-0.2582 \quad 2.0314 \quad-1.9087$
H $\quad 2.0744 \quad 1.0765 \quad-1.2899$
H $2.8406 \quad 2.9548 \quad 0.1677$
H $\quad 1.1751 \quad 4.3553 \quad 1.3683$
H $\quad-1.2268 \quad 3.8504 \quad 1.3538$
$\begin{array}{llll}\text { O } & -0.4901 & 0.1457 & -1.2769\end{array}$
AT $\quad 0.1503 \quad-1.4757 \quad 0.1168$

O-C interaction, $\Delta G_{298}^{*}=+3.3 \mathrm{kcal} \mathrm{mol}^{-1}$
N $\quad 2.2382 \quad 2.3236 \quad-0.0749$
$\begin{array}{llll}\text { C } & 0.9611 & 2.5921 & 0.0804\end{array}$
C $\quad 0.0112 \quad 1.4151 \quad 0.1239$
$\begin{array}{llll}\text { C } & -1.4131 & 1.7671 & -0.2017\end{array}$
C $\quad-1.8457 \quad 3.0456-0.1298$
$\begin{array}{llll}\text { C } & -0.9063 & 4.1104 & 0.1413\end{array}$
$\begin{array}{llll}C & 0.4466 & 3.9083 & 0.2494\end{array}$
H $\quad 2.5105 \quad 1.3670 \quad-0.2873$
H $2.9476 \quad 3.0469 \quad-0.0472$
$\begin{array}{llll}\mathrm{H} & -0.0330 & 1.2348 & 1.2321\end{array}$

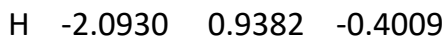
$\begin{array}{llll}H & -2.8937 & 3.2957 & -0.2893\end{array}$
$\begin{array}{llll}H & -1.2888 & 5.1299 & 0.2164\end{array}$
H $\quad 1.1321 \quad 4.7485 \quad 0.3603$
$\begin{array}{lllll}\text { O } & 0.5869 & 0.3343 & -0.5483\end{array}$
$\begin{array}{llll}\text { AT } & -0.0561 & -1.7178 & 0.0123\end{array}$ 